\title{
TOWARDS A NEW APPROACH OF DATA DISSEMINATION IN VANETS NETWORKS
}

\author{
Ouafa Mahma $^{1}$ and Ahmed Korichi ${ }^{2}$ \\ ${ }^{1}$ Department of Computer Science and Information Technology, Kasdi Merbah \\ University, Ouargla, Algeria \\ ${ }^{1}$ mahma.wafa@univ-ouargla.dz, mahma.wafa@gmail.com \\ ${ }^{2}$ Department of Computer Science and Information Technology, Kasdi Merbah \\ University, Ouargla, Algeria \\ ${ }^{2}$ ahmed.korichi@univ-ouargla.dz
}

\begin{abstract}
In the 2000s, ad hoc networks was developed and highly used in dynamic environment, particularly for inter-vehicular communication (VANETs : Vehicular Ad hoc Networks).

Since that time, many researches and developments process was dedicated to VANET networks. This was motivated by the current vehicular industry trend that is leading to a new transport system generation based on the use of new communication technologies in order to provide many services to passengers, the fact that improves the driving and travel's experience.

These systems require traffic information sharing and dissemination the example as the case alert message emitting allowing the driver to minimize driving risks. Sharing such information between vehicles helps to anticipate potentially dangerous situations, as well as planning better routes during congestion situations.

In this context, we are trying in this paper to model and simulate VANET Networks in order to analyze and evaluate security information dissemination approaches and mechanisms used in this type of networks in several exchanges conditions.This in order to identify their limitations and suggest a new improved approach. This study was conducted as part of our research project entitled "Simulation \& VANETs", where we justify and validate our approach using modeling and simulation techniques and tools used in this domain.
\end{abstract}

\section{KEYWORDS}

VANET, data dissemination, simulation, modeling, analysis and performance study.

\section{INTRODUCTION}

Today, road safety has become one of the biggest Challenges in the world, especially with the high dependence of people on vehicles and the growth of traffic problems (congestion and accidents ...). This reason push the researchers to Find ways for mitigate these problems. Among these ways : the intelligent transportation systems (ITS) developed new systems based on the

David C. Wyld et al. (Eds) : CCSEA, CLOUD, DKMP, SEA, SIPRO - 2016

pp. 13-23, 2016. (C) CS \& IT-CSCP 2016

DOI : $10.5121 /$ csit.2016.60202 
emergence of new communication technologies in the automotive industry in order to provide passengers more services to enhance the driving experience and travelers.

These systems recently known as Vehicular Ad hoc NETworks (VANET), systems allow communications between vehicles in order to exchange and share relevant information in the form of different categories of applications. A safety application is one of these applications that have aroused great interest among researchers.

In addition, the VANET systems often require knowledge of road conditions such as road status, accidents and congestion situations, and therefore the transmission of warnings messages allowing the driver of the drivers, this for minimize the dangers of driving. The exchange of such information and its sharing with distant vehicles can also help to anticipate potentially dangerous situations and to plan better routes during congestion situations.

The objective of this paper is to build a simulation model for the analysis of data dissemination protocols in vehicular ad hoc networks, in order to propose a new approach of traffic data dissemination which remedy insufficiencies of the approaches currently used. Where we interest in warning messages delivered in VANET networks. For achieve a better diffusion of these messages, consequently try to find the best parameters as time of dissemination and network overhead rates.

\section{VANET NETWORK}

Vehicular networks are a new class of wireless networks that have emerged by means of advances in wireless technology and the automotive industry. These networks also known with name of VANETs, which are considered as one of the real applications of ad hoc network, for communication between adjacent vehicles also between vehicles and stationary equipment.

The objective of VANET networks is to apply some notifications, such as dissemination of alert messages, reporting an accident between vehicles to reduce the probability of collision, the multimedia real-time applications and many other applications...

\section{SHARING AND DISSEMINATION OF DATA IN VANET}

Dissemination of traffic information is the principle of several research works, in consequence of the fact that the information is always shared concerns the traffic situation (for example the state of the road, condition of the car ...) in order to facilitate the movement of drivers and passengers on the roads, to enable them to take appropriate decisions to changes occurring in the road. In particular the information relevant to the risks and dangerous cases occur on the roads, this information necessities the immediate release and quick $[1,2,3,4]$ to ensure traffic safety.

This type of information is usually sent to a group of vehicles where the public interest here is consequently the most appropriate method is broadcast. although there are special cases where interest is concerned a specific group (eg: Geocast) (see Figure 1 and 2) .in general, the authors focus on methods Broadcast and Multicast, Geocast ... [1, 5, 6] according to their objective and their needs. In parallel with the fact that VANETs networks are mainly based on the ad hoc shortrange communication between the vehicles to improve safety in vehicular environments (WAVE: iEEE 802.11p). 


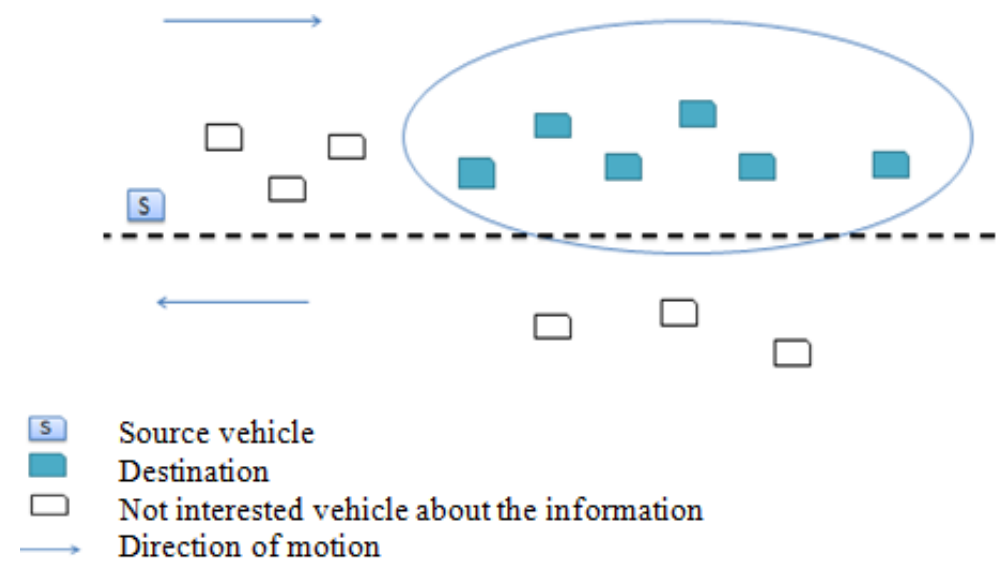

Figure 1. The information addressed to a specific group of vehicles
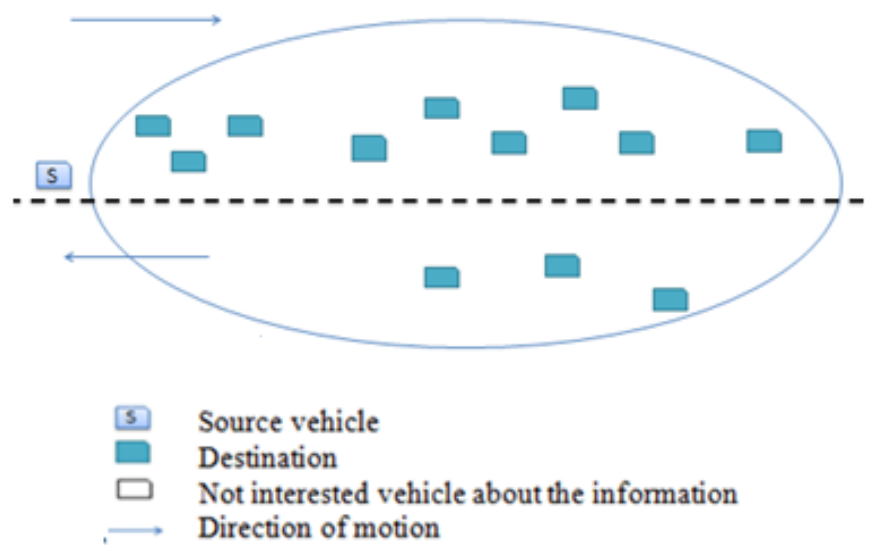

Figure 2. Information sent to all vehicles

Indeed, these previous studies use different concepts to achieve their goals and again to get the best results by a specific need (objective work). What is generally similar in these works is (1) the use of length of time. These shared information (warning messages, alerts messages ..) are issued only for a period of time (as long as the danger has existed), although there are very dangerous events cannot stand the wait and require the immediate release, this idea is not entirely absent, though his realism that is somewhat missed. (2) The lack of attention to the nature of the danger, for example:

- Loss of control over a truck carrying a flammable liquid, resulted in this slippage vehicle on Highway contains dozens of vehicles where the movement is very fast (vehicle speed can exceed $100 \mathrm{~km} / \mathrm{h}$ ), this shift is $100 \mathrm{~m}$ from the place of the accident (in Algeria).

- Another example: an accident occurs several times in European pays (like Belgium), the loss of control of the vehicle because of a breakdown on the level of the device named the limiting device and speed regulator where the vehicle was blocked at the speed of $200 \mathrm{~km} / \mathrm{h}$ on the road. 
- The results of these accidents were catastrophic, and that because of the lack of availability of the diffusion of this information in real time on the road.

Thus our objective in this work is not far from the mentioned points, we propose a communication system Geocast inter vehicles that enable the dissemination of safety messages (warning) in real time. Our objective is not the maintenance of the diffusion, but the reception and to send it message in time (in real time) potentially possible. This innovation based on the dissemination of the message of warning in the form of an alarm, in order to ensure a security service.

\section{SIMILAR WORK}

\subsection{Urban Multi -hop Broadcast (UMB)}

This protocol proposed in [7] designed to solve problems: Broadcast Strom, hidden nodes and which are related to the massive distribution in urban environments. This UMB protocol does not guarantee the absence of collusion, since it is possible to exist in more than one vehicle in the same segment. They can send in the same time the CTB. Again, this protocol works by some form of wait can be very long to select the next transmitter.

\subsection{Smart Broadcast (SB)}

It is a UMB improvement, wait time to assign the next re-broadcaster (relay) . with a delay function (WR), the farther vehicles from the source always have a waiting period when this latter is finished the vehicle transmitted the packet CTB to the source. once the source successfully receives this packet, it then transmits the data packet. This SB protocol as it is Indicated, it is an improvement of UMB at latency term .a shorter waiting period that is in UMB.

\subsection{Position-Based Adaptive Broadcast (PAB)}

The authors of SB propose the PAB protocol (Position-Based Adaptive Broadcast) [8]which is based on the waiting time for relay vehicle before forwarding the information to improve the techniques proposed for the facility access and efficiency of broadcasts. Contrary to SB which works at the distance between transmitter and receiver, the PAB uses a formula calculated by the vehicle position and speed to find the delay time value.

\subsection{Efficient Directional Broadcast (EDB)}

This protocol [9] is based on the direction one using bidirectional antennas of the vehicles. It works in same principle UMB. To solve the problem of retransmission number redundant latency is proposed for each node in the sector the emission before the retransmission, this time is different for each vehicle, it is calculated according to the distance between the vehicle and the transmitter.

\subsection{Reliable Method for Disseminating Safety Information (RMDSI)}

The authors in [10] the authors selected the delay time to differentiate the priority of retransmission for each vehicle. It is to function similarly with the previous protocol, when the vehicle receives a packet, a waiting time before rebroadcast it. After the expiration of the waiting time, the vehicle retransmits the packet. Vehicles intending to duplicate the rebroadcast before their waiting time expires cancel their broadcasts. By simulation, when the network is highly 
fragmented, RMDSI powerful that the UMB, which does not take the problem of the fragmentation of the network.

\subsection{Reliable Broadcasting of Life Safety Messages (RBLSM)}

In [11] the priority in the selection of relay is given to the vehicle with the vehicle nearest to the transmitter. The reason moreover nearer is more reliable. For example, a closer vehicle is supposed to have an intensity of better received signal. This protocol also uses the use of the packages of order and RTB CTB. The performances of the protocol are evaluated by simulation; However, only latency of one hop is provided. [3][11]

\subsection{Multi-hop Vehicular Broadcast (MHVB)}

This protocol [12] is similar to the precedents; it waiting time-based, such as the furthest vehicle from the transmitter always have the shortest waiting time. This time is calculated by an allocation function is not explicitly stated. After the expiration of the waiting time, the vehicle retransmits the packet. The protocol can be detecting traffic congestion. As each vehicle uses the number of its neighbors and its speed as an indication of congestion. [3]

\subsection{Abiding Geocast (AG)}

It is a system geocast of communication inter vehicle for the dissemination of massages of warning in VANET network. This model is proposed in order to ensure the dissemination of warning in order to: increase the probability of access to all relevant vehicle and reduce the overhead. This system uses different notions of time and space. To ensure inform group of vehicles exist in a geographical area someone on a risk proximity. Of which, the first vehicle detects the risk starts broadcasting a warning to other vehicles to inform them of this dangerous situation. In this work the authors used different dissemination strategies: (1) To achieve the first goal, they used the vehicle as reverse relay (2) Second goal: to update a time Waiting dynamically active vehicle for the next release. [1]

\subsection{Optimal multi-hop broadcast protocol for vehicular safety (OCAST)}

This protocol is an optimization of alert message dissemination in the VANET network for secure roads. Optimality in terms of time and number of transmission: using a dissemination strategy exploits the vehicle as opposed relay based on waiting times. And to complete the coordination of reliable and efficient distribution, smart periodic broadcasts to effectively adapt to VANETs networks. [5]

\section{OBJECTIVES AND METHODOLOGY OF THE STUDY}

Our mains objectives in these papers - as we indicated - are:

- Study of different dissemination protocols used in VANET networks .

- Create a model of simulation for applied the comparison and analysis performance of two studies in many cases: two scenarios and several parameters by analyzing the simulation results and found parameters. 
- Provide an optimal solution for the dissemination of safety messages on the roads.

This is generally for measuring the flexibility of these systems accidents that may occur suddenly in roads whatever its nature. Because of the real measurements are not possible, we chose to use in this work the analytical study based on simulation results.

\section{Performance Evaluation}

This study is a result of comparison and analysis of two studies of performance in many cases two scenarios and several parameters by analyzing the simulation results and found parameters. An optimal solution inspired by these studies for time dissemination and diffusion rate.

Although there are other protocols in addition to previously indicated [14], [4] ...., we chose OCAST and AG since they use parameters give potentially effective results compared to other proposes .En even more the OCAST is already compared with other in [2].

\section{A. Metrics}

Parameters measured are the rate of informed vehicles before the risk area, and the probability of access to all vehicles concerned in two different cases of risk: (risk is located in a random manner (case 2) and others in a predictable manner for the system (Case 1)). for a short duration time and long duration of time.

By simulation tools exists, our model is not much different from the used in [5] with a simple change in parameters for measure the effectiveness of the two systems in case of changing of risk on the road. The found results can be quite sufficient to compare and study the performance of these two protocols. .Generally OCAST is an improvement of what studied.

Indeed, we have chosen to add these results broadcasts capabilities used in [5] to the Geocast capacity proposed in [1] to provide an improved system for the dissemination of traffic information. So our model is defined in the next section but realistic simulation results of our approach are defined in details in our next production with a new algorithm for the message diffusion process in VANETs, where we use the simulator OMNET ++ (Version: 4.3.1) with the traffic simulator SUMO (0.17.1) and VEINS (2.2) to achieve our results.

\section{B. Simulation}

In this party, we use OMNET ++ open source simulation library that is written in $\mathrm{C}++$. This discrete event simulator simulate both types of networks (wired and wireless), in which the different network nodes can communicate via messages. The OMNET ++ tool has several advantages over others: simple to use, easy to learn by means of its user interface and its generic structure. It allows to find and obtain clear results, detailed and in many forms, diagram and drawings. This simulator is flexible to make changes in the created simulation models and even reuse these models. And more recently, this simulator is widely used in VANET network simulation domain, in practically protocols simulation model related to the MAC layer. Consequently, we prefer to use this simulation tool to avoid several problems related to the implementation of the simulation model by other simulators, especially when a large number of messages exchanged and shared between network nodes. 


\section{B.1. Simulators information's}

Table 1. Informations about the Simulators.

\begin{tabular}{|c|c|}
\hline Simulator & Version \\
\hline OMNET++ & 4.1 .1 \\
\hline Sumo & 0.17 .1 \\
\hline Veins & 2.2 \\
\hline
\end{tabular}

\section{B.2. The simulation parameters}

To achieve the simulation scenarios, we chose the following parameters:

Table 2. Simulation parameters.

\begin{tabular}{|l|l|}
\hline \multicolumn{1}{|c|}{ Description } & \multicolumn{1}{c|}{ value } \\
\hline Transmission range $(\mathrm{R})$ & $250 \mathrm{~m}$ \\
\hline Straight road & $7 \mathrm{~km}$ \\
\hline Mac layer & IEEE802.11 \\
\hline Safety distance & $250 \mathrm{~m}$ \\
\hline Effect distance & $10 \mathrm{Km}$ \\
\hline traffic volume $\lambda$ & $200 \sim 700(\mathrm{veh} / \mathrm{hr})$ \\
\hline Speed mean(Smean) & $30 \mathrm{~m} / \mathrm{s}$ \\
Speed variation $\varepsilon$ & $5 \mathrm{~m} / \mathrm{s}$ \\
\hline \multirow{2}{*}{ location of the warning } & Case $1=0$ meters \\
& Case $2=$ variable \\
\hline simulation time & $3000 \mathrm{~s}$ \\
\hline Start time of the warning event & Case $1=400 \mathrm{~s}$ \\
& Case $2=$ variable \\
\hline
\end{tabular}

As we say, we chose the same simulation parameters with slight changes, this for confirmation of the results. Except the second case where we tried to stay away from the private of fixed location of event for this risk to measure the effectiveness of the two systems. We omit the start time of the warning event to let the system reach a stable state considering the distribution of vehicles over the road. For both scenarios, when the warning event occurs, the beginner of dissemination is at the location of the safety line.

\section{Results and discussion}

\section{Case 1:}

In figure 4: different traffic value (measured density 1 to 15 vehicles are informed to $100 \%$ before the risk area (both systems). But imperfection AG appeared in (Figure 3) before arriving at risk (after the security line) this rate is a little different (the existence of vehicles after they inform exceed the safety line): a rate $(>20 \%)$ and $(<40 \%)$ of vehicle are not inform in the low density network. But for OCast delivery rate of $100 \%$ for all densities. 


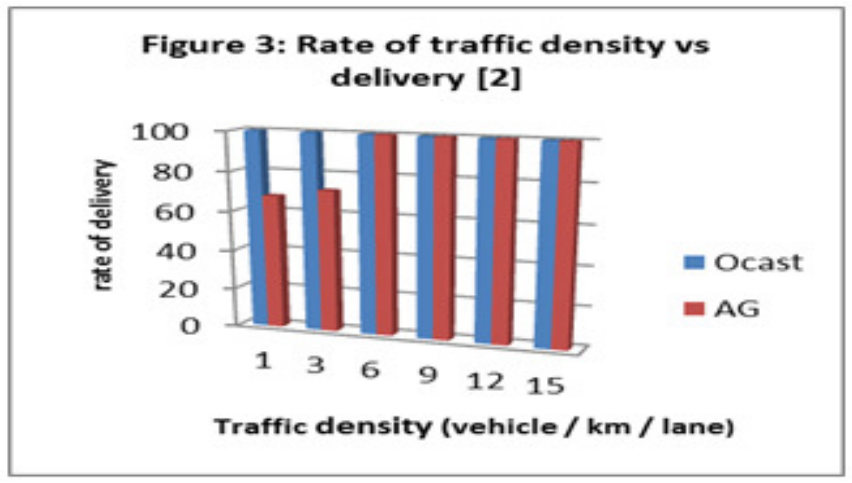

Figure 3. Rate of traffic density vs. delivery [5]

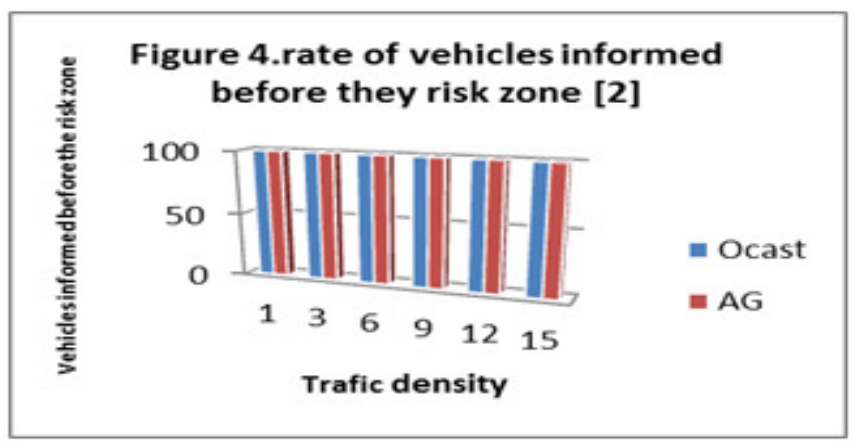

Figure 4. Rate of vehicles informed before risk zone [5].

\section{Case 2:}

In this case the simulation results are slightly different. In FIG 5: different traffic value (higher density then 7 vehicles are informed to $100 \%$ before the risk area (both systems).

But imperfection of both $\mathrm{AG}$ and Ocast system appeared in before arriving at risk (after the security line) and the beginning of the appearance of risk: the vehicles after they inform exceed the safety line, although this rate is not large but it is important in the warning information dissemination process.

Generally for all densities (Figure 3), OCast shows better performance than the AG as sending message "Stop" guarantee control the number of alerts issued in the network consequently it ensures minimization overload network.

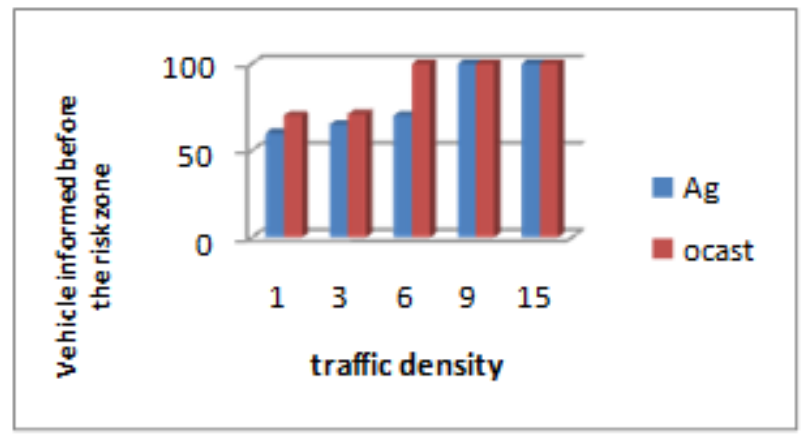

Figure 5.Rate of the vehicles before they risk area 
But the case of an unforeseeable risk, we see a better performance for both protocols in a density more than 6 , but this performance is decreased to the density less than 6 at the beginning of the appearance of risk.

We note that in the determination of the nature of risk and the results were good. Unlike in case the unknown risk (the location and characteristics) make traffic information sent uncertain and imprecise. Indeed, the simulation results show the effectiveness of OCAST compared to AG (case1) and but these results do not indicate the futility and uselessness of AG or OCast (case2).

Generally the purpose of our study is to measure the flexibility of these systems accidents that may occur suddenly in roads whatever its nature.

\section{Our proposed approach}

Our proposal is defined as the GODD "Geocast Optimization for Data Dissemination in vanet networks", a Geocast protocol introduced as an improvement time (the emission time) and number of transmissions (overhead), a flexible system to change the road for a better dissemination of safety data in VANET networks.

We try in this work as much as possible to ensure the immediate and optimal delivery of the warning information to all vehicles that are near to the risk, consider the validity of the message (the life time) and the direction of the mobility of vehicles in the network.

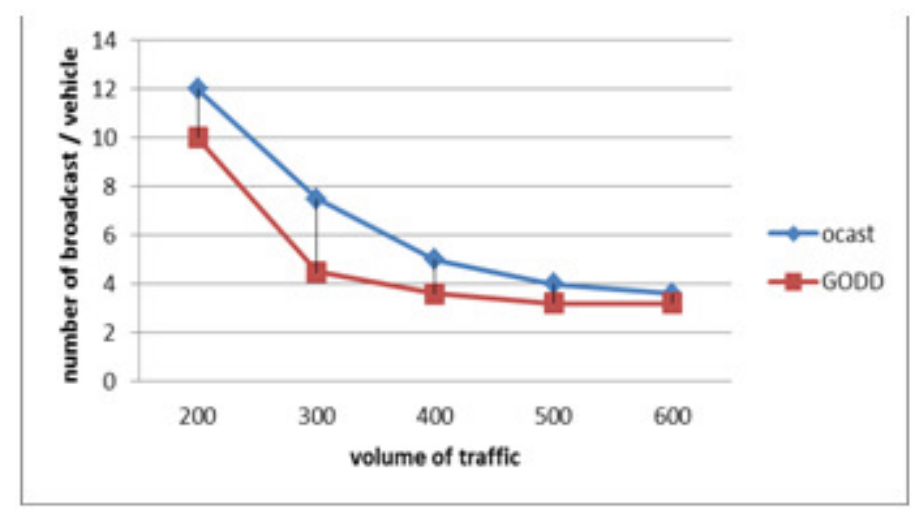

Figure 6. Rate of the vehicles before they risk area

This communication system Geocast inter vehicles is interested in the warning information released as soon as possible quickly the source node to several recipients in a region geographically define to secure these vehicles.

We use strategies to achieve our objective, to inform all these points while reducing unnecessary broadcasts and ensure a favorable reception time.

Broadcasts are organized in this system by the following process:

1- Initialization: when a risk occurs, the damaged vehicle will broadcast a warning message to neighboring vehicles (in its transmission area).

2- Selection of vehicle drivers: 
The relay vehicle is an active vehicle chosen by its proximity to the transmitter and its relative position in the latter.

3- Exit the system: The made to select a new relay, the source must receive a message of "stop" this relay to indicate you can leave the area.

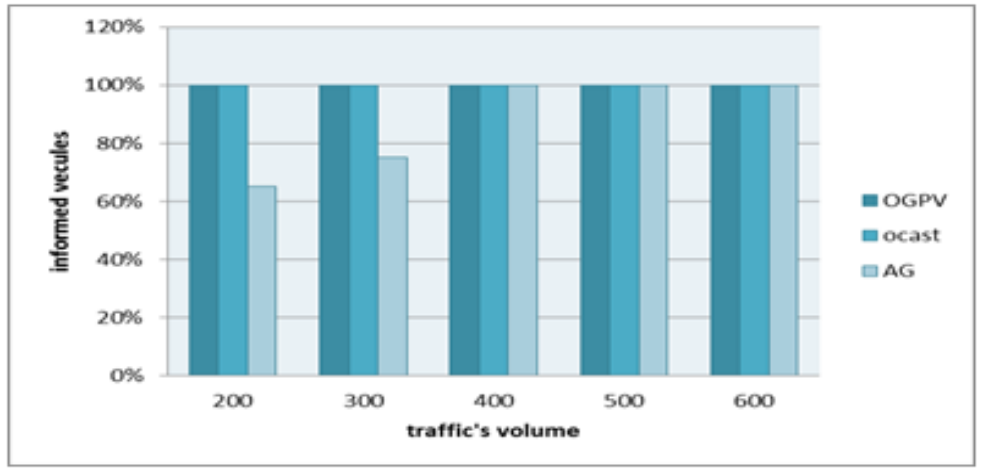

\section{CONCLUSIONS}

We tried to present in these papers an inter vehicle communication system Geocast: GODD as an optimization of the proposed approaches. Which we introduce a new contribution to more secure passengers in the roads.

This work is a description of what our approach detailed in our next production, where we show the optimality of the system by the simulation tool by OMNET ++ simulator used (version 4.3.1) and SUMO (Version 0.17) with VEINS.

The system offer better message delivery performance side of caution, the thing that evolve road services for VANETs.

\section{REFERENCES}

[1] Y.Qiangyuan Yu and H. Geert (2008) “Abiding Geocast for Warning Message Dissemination in Vehicular Ad Hoc Networks", IEEE International Conference on Communications Workshops (ICC) Beijing, China, pp. 400-404.

[2] M. Koubek, S. Rea, D. Pesch (2008) "Effective Emergency Messaging in WAVE based VANETs" Centre for Adaptive Wireless Systems,Electronic Engineering Dept, Cork Institute of Technology, , in Proc. First International Conference on Wireless Access in Vehicular Environments (WAVE 2008), Dearborn, MI, USA.

[3] S. Panichpapiboon, and W. Pattara-atikom (2011)“A Review of Information Dissemination Protocols for Vehicular Ad Hoc Networks", IEEE.

[4] N. Haddadou (2014) " Réseaux ad hoc véhiculaires : vers une dissémination de données efficace, coopérative et fiable" (doctorate These ).

[5] A. Benaidja ,S. Moussaoui and F. Naït-Abdesselam (2013) "An Optimal Broadcast of Warning Messages in Vehicular Ad Hoc Networks ", International Journal of Computer and Information Technology , ISSN: 2279 - 0764, Volume 02- Issue 05. 
[6] C. Maihofer, T. Leinmuller, and E. Schoch (2005) "Abiding geocast: time-stable geocast for ad hoc networks", in Proceedings of the 2nd ACM international workshop on Vehicular ad hoc networks (VANET'05), New York, USA, pp. 20-29.

[7] G. Korkmaz, E. Ekici, F. Ozguner, and U. Ozguner (2004) Urban multi-hop broadcast protocol for inter-vehicle communication systems. In ACM International Workshop on Vehicular Ad Hoc Networks, New York, NY, USA.

[8] Y.T. Yang and L.D. Chou (2008) "Position-Based Adaptive Broadcast for Inter-Vehicle Communications," Communications Workshops, ICC Workshops '08. IEEE International Conference on , vol., no., pp.410-414, 19-23.

[9] D. Li, H. Huang, X. Li, M. Li, and F. Tang (2007) "A distance-based directional broadcast protocol for urban vehicular ad hoc network," in Proc. IEEE Int'l Conf. on Wireless Comm., Networking and Mobile Computing (WiCom), Shanghai, China, pp. 1520-1523.

[10] S. Khakbaz and M. Fathy (2008) "A reliable method for disseminating safety information in vehicular ad hoc networks considering fragmentation problem," in Proc. IEEE Int'l Conf. on Wireless and Mobile Communications (ICWMC), Athens, Greece, pp. 25-30.

[11] M. Taha \& Y. Hasan (2007)"VANET-DSRC protocol for reliable broadcasting of life safety messages," in Proc. IEEE Int'l Symp. on Signal Processing and Information Technology, pp. 104109.

[12] T. Osafune, L. Lin, \& M. Lenardi (2006) "Multi-hop vehicular broadcast (MHVB)," in Proc. IEEE Int'l Conf. on ITS Telecomm. (ITST), Chengdu, China, pp. 757-760.

[13] Q. Xu, T. Mak, J. Ko and R. Sengupta (2004) "Vehicle-to vehicle safety messaging in DSRC", in Proceedings of the First ACM workshop on Vehicular ad hoc Networks (VANET).

[14] Harshvardhan P. Joshi (2006) “ Distributed Robust Geocast:A Multicast Protocol for Inter-Vehicle Communication", Computer Networking - Electrical Engineering, Raleigh these.

\section{AUTHORS}

\section{Ouafa MAHMA}

Obtained on testimonies license (2006) and master (2011) in the fundamental automatic information from the University of Ouargla in Algeria .Prepare for a doctoral degree in the field networks and informatics systems in the same university.

\section{Ahmed KORICHI}

Obtained on testimonies doctorate (2004) in automatic information from the University of Batna in Algeria. Prepare for a professor degree in university of Ouargla in Algeria .

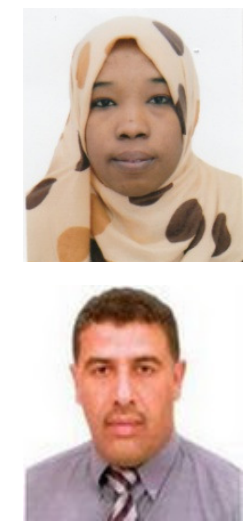

